\title{
PERSPECTIVES
}

OPINION

\section{Studying complex biological systems using multifactorial perturbation}

\begin{abstract}
Ritsert C. Jansen
High-throughput genomics, transcriptomics, proteomics and metabolomics have the potential to identify the functional consequences of induced and natural genetic variation. Surprisingly, the experiments of most genomics researchers still mainly involve perturbing a biological system of interest by modifying either one factor or one gene at a time. By contrast, this article argues that multifactorial experimentation would allow the study of many more biologically relevant questions in parallel at the same or lower cost.
\end{abstract}

The recent progress in genomics and genetics, particularly in the high-throughput measurement of DNA transcript and protein levels, has led to a surge of interest in the fields of SYSTEMS BIOLOGY and COMPLEX TRAITS BIOLOGY to study complex human diseases and disorders $\mathrm{s}^{1,2}$. Although such optimism is justified by the success that has been achieved in identifying the genetic basis of monogenic diseases and disorders, such as cystic fibrosis $3^{3,4}$ and Huntington disease ${ }^{5,6}$, unravelling how genes "talk to each other" is likely to be an intimidating effort for more complex traits and processes. This is also true when analysing the genetic actions/interactions that occur in experimental systems: even organisms that have $10^{3}-10^{4}$ genes, such as the budding yeast Saccharomyces cerevisiae, can produce more than $10^{4}-10^{5}$ gene products, which can easily account for more than $10^{5}-10^{7}$ interactions. Analysing how genes act and interact is complicated further by the number of pathways in which genes can function, the number of environmental triggers that act on these pathways and the number of alleles that exist for each gene. Progress in systems biology will depend on finding new ways to tacklethis higher-order complexity. A largescale study of transcript, protein or metabolite abundance in a perturbed system can provide the data for unravelling the complex interplay of genes and the environment. In this article, I discuss that experiments in which biological systems are perturbed and studied in a multifactorial manner - that is, in which gene variants and environmental factors are present in many combinations - are likely to bemore informative and economical than those in which biological systems are studied by varying one environmental factor or gene at a time. I explain what we can learn from multifactorial perturbation experiments and how this knowledge can be applied. It also becomes apparent that a multidisciplinary research effort, involving the increased input of statistics, mathematics and computing sciences, is crucial for the success of a multifactorial approach. The key question is how to obtain the greatest knowledge about complex biological interactions for thelowest possible cost, through clever experimental designs, models and methods of analysis. One issue that has yet to be resolved by the new multifactorial strategies is how complex are the traits and processes of interest and, consequently, whether systems biology will indeed substantially increase our knowledge of many human diseases and disorders. If successful, then human health might benefit, through the design of improved drugs and through the shift from population-based to patient-specific drug treatment, as well as drug or dietary restriction. In addition, the development of healthier and safer (for example, nonallergenic) food becomes a possibility, through the more informed breeding of plants, animals and microorganisms in our food chain.

\section{Perturbing biological systems}

Themost widely used strategies for perturbing a biological system use gene-knockout technologies, mutagenesis or transgenesis. Typically, these approaches involve altering one gene at a time; large collections of single-alteration lines have been produced as a result, such as in $\mathrm{S}$. cerevisiae ${ }^{7}$, and are shared by the research community. An example of a single-gene perturbation experiment is provided by Ideker et al. ${ }^{8}$, who studied the yeast galactose metabolic pathway, the molecular biology of which is well understood. Having defined the genes of interest (nine galactose pathway (GAL) genes) and an initial model to describehow these genes interact, the authors perturbed the galactose pathway by deleting each of the GAL genes in nine different lines. For each perturbed condition, the authors then carried out replicate hybridizations in four different DNA microarrays, to obtain robust estimates of how the gene expression profile of each knockout strain differed from that of the wild type (FIG. 1a). Any gene that is expressed equally in the two strains will have an expression ratio of $\sim 1$ (corresponding to a log-ratio of zero). The main advantage of this approach is that the analysis and interpretation of such experiments is straightforward. However, the genetic background remains constant and so the effect of the single alteration in another background is unknown. In the most extremesituation, this occurs when complex gene-gene interactions are involved, and 


\section{PER S PECTIVES}

a

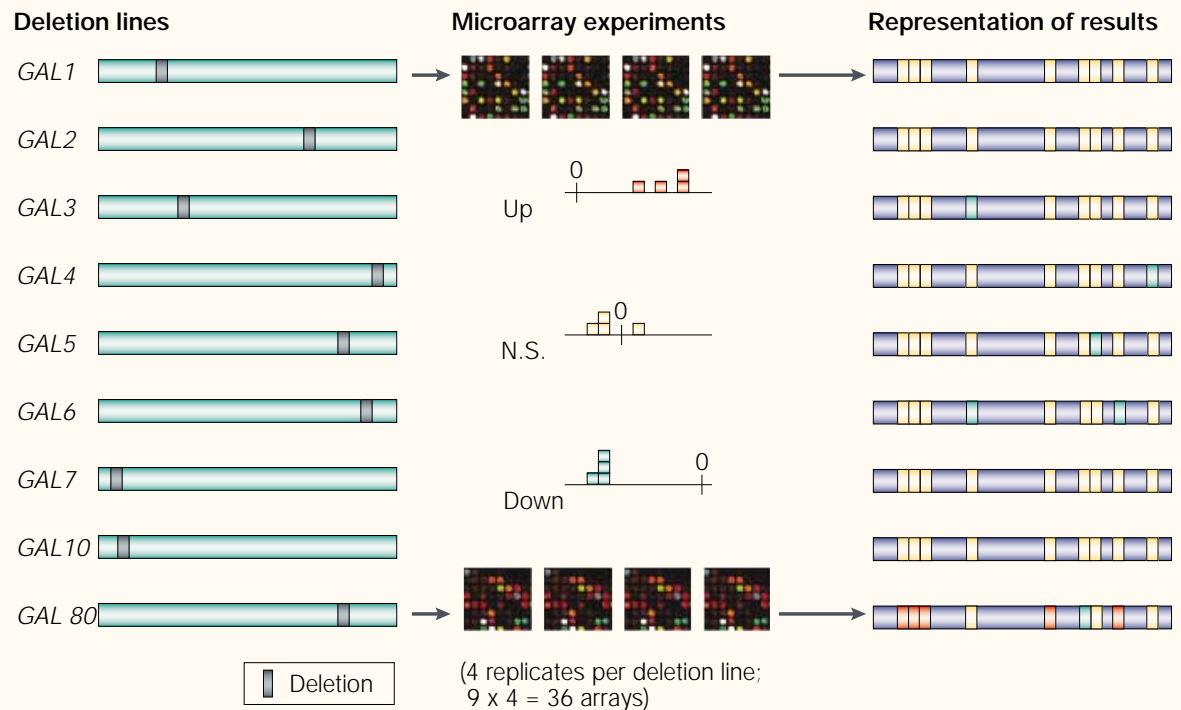

b
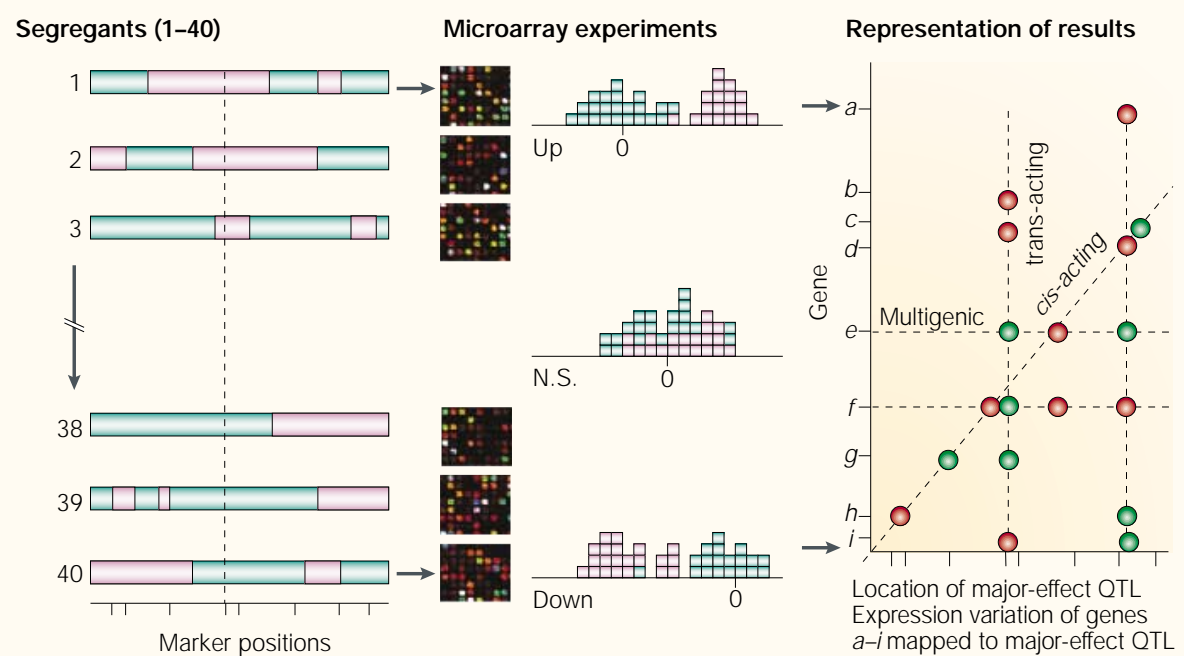

Figure 1 | Two strategies for perturbing biological systems. a | Gene knockout. An initial model for the interaction of nine yeast GAL genes is tested through expression profiling of these and many other genes in nine single-gene knockout lines. The nine genes are indicated as dark green boxes on the genome. Upregulation (red), downregulation (green) and no effect (yellow; N.S., not significant) are assessed through the statistical analysis of logarithm-transformed expression data that was obtained using four microarray replicates, in which the expression of GAL genes in each knockout strain was compared with that in the wild type. Several newly detected interactions refined the initial interaction model: for example, in the presence of galactose, lines in which GAL7 and GAL10 had been deleted had unexpectedly reduced the expression levels of the other GAL enzymes. Diagram based on Ideker et al. ${ }^{8} . \mathbf{b} \mid$ Genetical genomics. The genetic differences between two yeast strains are analysed through the expression profiling of 40 segregants obtained by crossing the strains. Parental alleles and genome blocks are indicated throughout by green and blue, respectively. The logarithm of the expression of each of 6,215 genes is analysed across the 40 segregants with software used to map QUANTITATIVE TRAIT LOCI (QTL). This method detects the 'major-effect' loci that underlie the observed genetic variation between the two parental strains by scoring either upregulation (Up) or downregulation (Down) in gene expression (N.S., not significant change). Several gene transcripts were found that map to two or more loci in each segregant. Variation in the expression of genes e and $f$, for example, maps to a similar set of loci (horizontal dotted lines), indicating that e and $f$ might function in the same genetic pathway. Major-effect loci can be cis-acting (dotted line at an angle) or trans-acting (dotted vertical lines). Diagram based on Brem et al. ${ }^{12}$. Red indicates upregulation, green indicates downregulation of gene expression. In their analysis, 570 gene transcripts showed differential expression in the segregating population and were mapped to one or more loci: $36 \%$ of the transcripts were cis-linked, $45 \%$ were linked to one of only eight trans-acting modulators. So, only a few of the major-effect loci were trans-acting modulators of many genes, but they accounted for a large fraction of all segregating transcript levels. any conclusion drawn from perturbing a gene in one particular background might be completely irrelevant for other backgrounds. The same principles apply to any other strategy that is based on perturbing a single gene at a time, particularly singlegene transgenesis and mutagenesis (see REFS 9,10 for further examples of single-gene perturbation experiments on sea urchin and mouse, respectively).

Several research groups have recently started to study systems that have been perturbed through genetic crosses, a process that is known as GeNETICAL GEN OMICS ${ }^{11}$. This approach has been successfully applied to $\mathrm{S}$. cerevisiae ${ }^{12}$, mouse $^{13}$ and Drosophila melanogaster ${ }^{14}$. In the genetical genomics approach, the genetic mechanisms of segregation and recombination are used to reshuffle the genomes of two or more donor parents, to produce a population of segregating offspring with many combinations of gene variants. One illustrative example is provided by Brem et al. ${ }^{12}$. These authors exploited the natural genetic variation that exists in S. cerevisiae by crossing a standard laboratory strain (BY) with a wild isolate from a California vineyard (RM). They generated 40 segregating offspring and profiled each offspring on one expression microarray relative to the reference BY strain (the segregants were also profiled a second time, in which the fluorescent dyes were swapped) (FIG. 1b). For each segregating gene, an average of 20 segregants inherit the wild-type RM allele, while theother 20 inherit the laboratory-type BY allele. The latter group might express genes at the same level as the parental BY strain used as the reference, in which case the corresponding gene expression ratios should be $\sim 1$ (zero on a logscale). The difference in expression between the parental RM and BY strains can be assessed in two ways: by testing whether the log-ratios of the gene expression levels of the 20 segregants that carry a particular RM allele significantly depart from zero compared with those of the reference BY strain; or by using all segregants and testing whether the log-ratios of gene expression levels seen in the $20 \mathrm{RM}$-carrying segregants differs significantly from the 20 that carry the particular BY allele. It is important to note that significant differences in expression between the 20 segregants that carry a particular BY allele and the reference BY strain highlight the existence of (complex) interactions between the gene in question and its genetic background. Brem et al. ${ }^{12}$ showed that the log-ratios of the expression of two genes (YLL007C and XBP1) between 
the BY-carrying strains and the BY reference are close to zero, indicating the absence of major interactions between the gene and the genetic background (gene $\times$ genetic background interactions).

In the next section, I focus on refinements of the genetical genomics method. Thereafter, I evaluate the relative merits of genetical genomics versus single-gene perturbation strategies.

\section{Refining genetical genomics}

The effectiveness of the basic genetical genomics approach can be improved in several ways. AsI describe in the following sections, these improvements involve carefully evaluating the possible experimental designs (for example, choosing the type of segregating population that is most appropriate for unravelling complex interactions), the possible models (such as those that take into account relevant biological or technical sources of variation) and the methods of analysis (simpler methods might outperform more exhaustive ones in some cases).

Choosing an appropriate population. The type of segregating population that is used in a genetical genomics analysis can improve the power and efficiency of this type of strategy. For example, Demant and $\mathrm{Hart}{ }^{15}$ proposed the use of RECOMBINANT CONGENIC STRAINS (RCSS) to analyse genetic traits that are determined by more than one gene in the mouse. They generated RCSs in which the fraction of the genome derived from the donor strain was $\sim 12.5 \%$. The RCS that showed the largest phenotypic difference compared with the recipient strain was used to generate a larger segregating population, so yielding perturbed lines that were only segregating for more relevant genomic areas ${ }^{16}$. Other types of population, SUCh aS RECOMBINANT INBRED LINES (RILS) and ADVANCED INTERCROSS LINES, have been proposed and applied successfully ${ }^{17}$. Recently, the use of CHROMOSOME SUBSTITUTION STRAINS (CSSS) instead of RCSs has been advocated ${ }^{18}$ (see FIG. 1 in REF. 18 for a graphical comparison of RCSs and CSSs). Although dealing with one chromosome at a time is a simple and appealing strategy, it could be a less efficient way of studying multigenic traits: unravelling the effects of linked genes is hampered by the shortage of recombination events, making it difficult to resolve statistically the effects of linked genes. The debates about the relative merits of RCSs, CSSs and other types of segregation population are ongoing. For example, The Complex Trait
Consortium ${ }^{19}$ (see onlinelinks box) is now considering the construction of recombinant lines that result from an eight-way cross between eight selected mouse strains. These strains would be useful to study the effects of a large proportion of the existing natural allelic variation in a broad range of genetic backgrounds. A further option in designing a multifactorial experiment is to exploit the information contained in two or more populations ${ }^{20}$. For example, simultaneously analysing segregating populations derived from crosses $A \times B, B \times C$ and $A \times C$ between three inbred parents $A, B$ and $C$, allows inferences to be made efficiently about gene $\times$ genetic background interaction. For each gene under study, the differential expression between the two gene variants $A$ and $B$ can be tested in the progeny of the cross $A \times B$, between $B$ and $C$ in the progeny $B \times C$ and between $A$ and $C$ in the progeny of $A \times C$. Only in the absence of interactions will the differential expression seen between $A$ and $B$ in the $\operatorname{cross} A \times B$ and the differential expression between $B$ and $C$ in the cross $B \times C$ sum to the differential expression between $A$ and $C$ in the cross $A \times C$.

Generating biologically relevant models. Models should relate the (biomolecular) phenotype to the genotype and environment in a biologically meaningful way. These considerations can fail to be accurately incorporated into the study of complex multifactorial systems for two reasons. First, important sources of biological variation and noise might be missed. Second, although all relevant factors might be included in the model, some assumptions might be wrong. As a result, the effects that are of biological interest might be masked, or effects that have no biological basis might be reported as significant.

It is well known that genomic factors such as locus- and gene-copy number, chromatin structure, promoter and operon structure, al ternative splicing, methylation and transcriptional hot spots - all have an important and quantitative rolein controlling

\section{Glossary}

\section{ADVANCED INTERCROSS LINES}

Subsequent generations ( $F_{3}, F_{4}$, and so on) of an

intercross pedigree that are used for the high-resolution mapping of trait loci.

CHROM OSOME SUBSTITUTION STRAIN

(CSS). Each CSS contains an entire chromosome of a donor parent placed in the genetic background of the recipient parent.

COMBINATORIAL PARTITIONING

A computational strategy that consists of pooling genotypes from multiple loci into a smaller number of classes, thereby avoiding the increased dimensionality that is associated with modelling interactions between loci or between loci and the environment.

\section{COM PLEX TRAITSBIOLOGY}

The study of traits that are determined by many genes, which almost always interact with environmental factors.

\section{EPISTASIS}

In the context of quantitative genetics, epistasis refers to any genetic interaction in which the combined phenotypic effect of two or more loci is less than (negative epistasis) or greater than (positive epistasis) the sum of the effects at individual loci.

GENETIC ALGORITHM

A numerical optimization procedure that is based on evolutionary principles such as mutation, deletion and selection.

GENETICAL GENOMICS

The process that uses gene expression profiling and marker-based fingerprinting of each individual in a segregating population to analyse the cis- and trans-acting factors that underlie variation in gene expression. Thisinformation can then be used to reconstruct a gene network.
MARKOV CHAIN MONTE CARLO STRATEGY A randomized computational approach for identifying the most likely among many possible models.

MULTICOLLINEARITY

The situation in which two or more predictors (or subsets of predictors) are strongly (but not perfectly) correlated to one other, making it difficult to interpret the strength of the effect of each predictor (or predictor subset). For example, it would behard to detect a geneif its effect is 'absorbed' (or masked) by combinations of genetic background action/interaction parameters in the model.

QUANTITATIVE TRAIT LOCI

(QTL). Genetic loci or chromosomal regions that contribute to variability in complex quantitative traits (such as plant height or body weight), as identified by statistical analysis. Quantitative traits are typically affected by several genes and by the environment.

RECOMBINANT CONGENIC STRAIN

(RCS). A population of fully homozygous individuals, each of which contains a restricted part of one of the two genomes from which the inbred lines were created.

RECOM BINANT INBRED LINES

(RILs). A population of fully homozygous individuals that is obtained through the repeated selfing of an $F_{1}$ hybrid, and that comprises $50 \%$ of each parental genome in different combinations.

SYSTEMSBIOLOGY

The study of the complex interactions that occur at all levels of biological information - from whole-genome sequence interactions to developmental and biochemical networks - and their functional relationship to organism-level phenotypes.

VARIANCE

A statistic that quantifies the dispersion of data about the mean. 


\section{PER S PECT IVES}

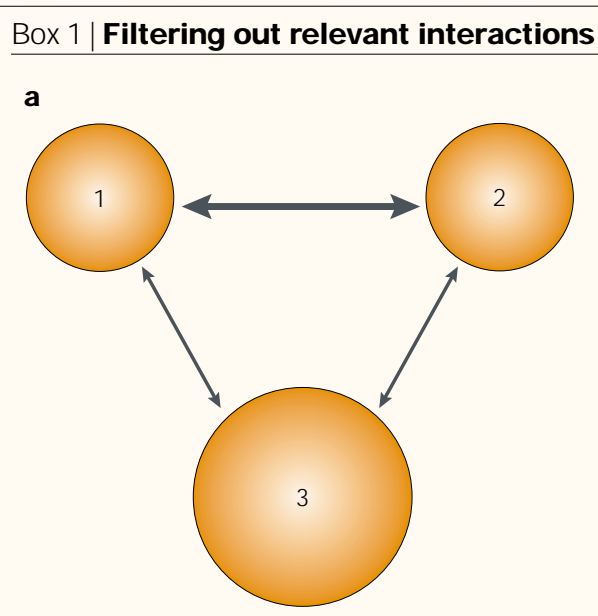

b

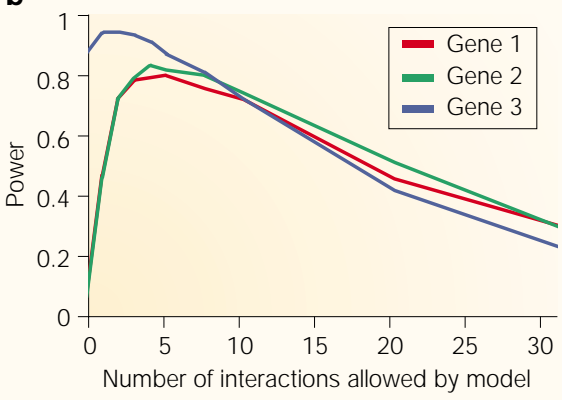

The filtering of biologically relevant genetic interactions is difficult as we must search in two or more dimensions for all possiblegene-gene or higher-order interactions. H owever, simplified searches (those that allow for a smaller number of interactions in the model) might work sufficiently or in some cases better than full searches, as shown in the following example (based on REF. 34).

Panel a shows threegenes (1-3), which together encode a hypothetical biochemical compound. Genes 1 and 2 interact strongly (indicated by thethick arrow), showing positive and negative EPISTASIS for different allele combinations, while the main effects at the individual loci are relatively small (indicated by small orange circles). Gene 3 interacts weakly with genes 1 and 2 , but the main effect of substituting oneallele of gene 3 by another allele is relatively large. The genes are located on an imaginary genomethat has threechromosomes, each of which consists of 100 centiMorgans ( $\mathrm{CM}$ ) and is covered by 33 markers, with $10 \mathrm{cM}$ between adjacent markers. The example is designed to illustrate the ability of

different models to predict the presence of thethree genes. The analysis was based on 1,000 independent data sets that were generated by computer simulation. A data set consists of trait and marker scores for 200 backcross individuals between two homozygous inbred strains that carry different alleles for the three genes under study.

All 1,000 data sets were analysed using the quantitative trait loci (QTL) analysis software EpiM QM (REF. 34), ignoring any previous knowledge of the number of genes involved. EpiMQM searches for QTL in a one-dimensional genome search: at each map position under study, the model considers the main effect of the putativeQTL, the main effects of a variablenumber of markers used as cofactors (to model the effects of genetic background), and the effects of a variablenumber of interactions between the putative QTL and the marker cofactors (to model interactions between the putative QTL and the genetic background). The power to detect a gene depends on the number of interactions that areallowed for in the model (plotted on thex axis in panel b). Thegraph shows that genes 1 and 2 have low power to be detected if the number of interactions allowed by the model is zero, which is not surprising as these genes have small main effects. Luckily, a small increase in the number of interactions greatly improves the power of detecting them. Any further increase in the complexity of the model, however, decreases the power to detect interactions, due to the need to test so many gene $\times$ genetic background interactions and due to the increased chance that the effect of a gene is 'absorbed' (or masked) by combinations of genetic background action/interaction parameters in the model. Panel b is modified with permission from REF. $34 \odot$ (2002) TheGenetics Society of America.

variation in gene expression (although this is often not completely understood at the physical level). Environmental factors, such as the time and place at which the biological sample is collected, are also crucial. For example, transcript and protein abundance often do not correlate as well as expected, which could be taken as evidence for posttranslational modifications $s^{8,21}$. H owever, a low transcript-protein correlation (for example, that observed in REFS 8,21) can equally well be explained as arising from different sources of systematic and random errors that are associated with the different sampling and processing of RNA and protein ${ }^{22}$. So, overlooking important biological and technical factors can lead to conclusions that might be correct, but that are not proved on the basis of the experimental evidence

A second source of error derives from the assumptions that are incorporated into the analysis. It is important to realize that genetic concepts, such as additivity, dominance and
EPISTASIS, depend on the scale at which data is measured and/or analysed, the choice of which therefore needs careful consideration ${ }^{23}$. Some high-throughput techniques score the absolute amount of expression product (for example, see REF. 24), whereas other techniques (such as microarrays) can 'only' measure the relative amount of product from a target sample compared with a reference sample. In some cases, the absolute amount of gene product is simply the sum of the contributions of the individual effects of the alleles involved (see REF. 25 for an example in tobacco plants). However, formulating genetic models for the logarithm-transformed data (as opposed to the original, more biologically relevant scale) might reveal non-additivity of allelic effects and, therefore, might lead erroneously to the conclusion that the alleles in question interact. So, the modelling of the interplay of many genes - which is the aim of complex systems biology - is not without danger. Any model can bewrong (almost by definition), but particularly complex (overparameterized) models have much flexibility to hide their lack of biological relevance. From this perspective, the pros and cons of the methods that measure and analyse absolute or relative amounts of gene product need to be evaluated in more detail before a biologically relevant model can be selected.

Even though the ultimate goal is to model the complex interplay between many factors, varying a single or a few factors of interest in 'simple' transgenic studies can still be informative for understanding the factors that underlie variation in gene expression (for example, see REFS 26,27). These 'traditional' molecular biology experiments might be more important for making progress in systems biology than is appreciated at present. A better understanding of expression variation will lead to experimental designs that take previously ignored factors into account in the analysis, with the benefit of improving the power to detect the differential expression of genes of interest and increasing the chances of drawing biologically meaningful conclusions.

Methods of analysis. Two challenges must be confronted when analysing gene interactions: unravelling the complex interplay between the genes that underlie the phenotype under study; and, having done so for thousands of expression phenotypes, putting the pieces of the puzzle together to reconstruct the gene networks. These two issues are discussed below. 
A principal concern in the analysis of a particular expression phenotype is how to deal with the relatively low number of individuals in the population compared with the largenumber of genes and their interactions (known as 'predictors'). This is known in computational science as the ' $n \times P^{\prime}$ ' problem ( $s$ all $n$, number of individuals, and high $P$, number of predictors). The aim is to find all the loci that underlie the variation in expression of a gene under study. This type of analysis requires a large degree of computational complexity, as it involves searching across all subsets of interacting genes. Furthermore, even if this were a tractable problem, the statistical power of any analysis is diminished by theneed to test many combinations of interacting genes and by the potential MULTICOLLINEARITY between subsets of predictors. A relatively small increase in the number of genes can encodea huge increase in the complexity of an organism. For example, with two states per gene (for example, on-off), humans, which have $\sim 30,000$ genes, can encode $2^{30,000}$ multigenic states, whereas nematodes, which have $\sim 20,000$ genes, can have'only' $2^{20,000}$ states, the ratio of complexity being about $10^{3,000}$ in favour of humans ${ }^{28}$. An exhaustive search across all subsets of interacting genes is not feasible, but various computational techniques that are derived from mathematics, statistics and informatics can hel $p$ to filter out theimportant interactions and to exclude the unimportant ones. For example, clever optimization techniques that use GENETIC ALGORITHM S $^{29}$ or MARKOV CHAIN MONTE CARLO STRATEGIES ${ }^{30}$ can beused to browse through themany models that are defined by different sets of genes and geneinteractions. These approaches modify and improve the current model step-by-step, so that we do not seetrapping into 'locally' optimal models. Although the efficiency of thesemethods might indeed be comparablewith that of an exhaustive search, translating the results into meaningful biological conclusions can still be difficult, and the danger of over-interpreting the results and of obtaining non-replicableconclusions is always present. Therefore, it is probably wise to use these methods only in combination with others that restrict the type and the number of interactions and limit the size of their effects, to reduce search complexity and the number of statistical tests hopefully in a biologically acceptable way. Collapsing multilocus genotype categories into fewer classes by using COM BINATORIAL PARTITIONING methods, thereby reducing the number of parameters, can reduce computational complexity. This method has been applied to find higher-order gene

\section{Table 1 | Pros and cons of two types of perturbation strategy \\ Using natural genetic variation (such as genetical genomics) \\ Using engineered variation (such as point}

Pros

Multifactorial in concept: powerful and cost effective

Broad applicability of results: gene variants (alleles) are tested in many genetic backgrounds

Allows the discovery of subtle effects obscured in engineered variation

Variation in stocks available at no extra production and maintenance cost

Allows the identification of hypothetical connections between major-effect loci and the variation in expression of the genes that they affect

Gives statistics on the number of genes acting in cis or in trans, the size of gene actions/interactions, and so on

No initial model of gene network needed

The only strategy available to perturb human systems

\section{Cons}

Cannot detect genes for which there is only a single allele in the population Possibly restricted applicability of results

Higher complexity due to the polygenic nature of (biomolecular) phenotypes in the case of whole-genome segregation Possibility of increased noise due to unexplained genetic variation

Narrowing down from QTL to candidate gene might be difficult

QTL, quantitative trait loci. interactions in human population studies ${ }^{31,32}$. I gnoring higher-order interactions between three or more genes is another strategy to reduce computational complexity. This method has been used widely (for example, see REF. 33 for a lung cancer study in the mouse). Searching for interactions between a putative gene and other genes in the genetic background - while ignoring interactions among genes in the genetic background - is an even more stringent strategy ${ }^{34}$. A one dimensional genomescan, which tests for candidates that interact with a putative gene by conceptually moving it along the chromosome, can efficiently highlight regions of interaction (BOX 1). In general, the computational problems are much harder to solve for non-experimental biological systems, because the multifactorial complexity cannot be tested by appropriate experimental designs (instead, we have to make use of natural populations, existing pedigrees, case-control studies, and so on, for which models are more difficult to test).
Perturbs a single gene at a time: not powerful and not cost efficient

Distortion of gene function might obscure normal gene interactions in the affected pathway

Many deletions might be needed to locus to overcome buffering by the biochemical network

Leads to gene variants that have more easily detectable effects

Changes a gene with only one gene variant into a gene with two variants

Introduces qualitatively different proteins and thereby uncovers the range of gene function
The second challenge, network reconstruction, need not start from scratch if an initial network of molecular interactions has already been established. Previous hypotheses are a prerequisite for deciding which genes to disturb using knockout, transgenesis and directed mutagenesis. By contrast, previous knowledgeis not required for a genetical genomics strategy to be successful; therefore, this can be a useful hypothesis-free method for constructing gene networks. Although integrating observed expression profiles with a starting model is not an easy task, it is certainly easier than reconstructing a network from scratch (that is, without an initial model). For strategies that perturb one gene at a time, a method has been advocated that relies on a quantitative measure of how the expression of a gene responds to the perturbation of another gen $\mathrm{e}^{35,36}$. Stronger pairwise combinations are then depicted as connections in a gene network. $A$ genetical genomics strategy for putting the pieces of the puzzle together is based on theidea that genes that function in the same pathway (and the 


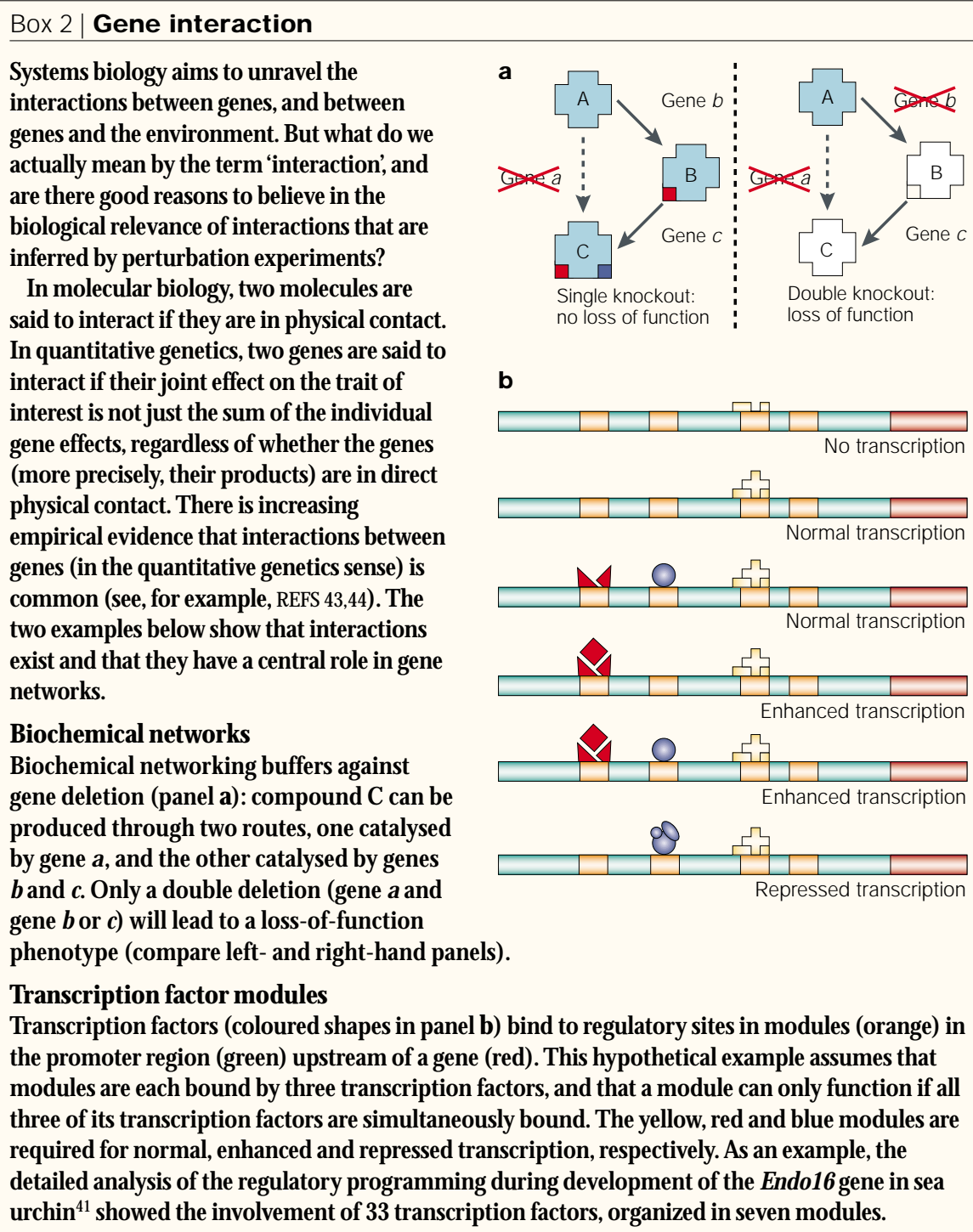

expression of which is therefore likely to vary in the same way) arelikely to map genetically to similar regions on the genomeand that cisacting genes are good candidates for quantitative trait loci (QTL) $11,12,14$ (seeal so FIG. 1b).

It is important to note that this strategy is not restricted to the analysis of expression traits: any trait, whether genomic or phenotypic in origin, can beintegrated. Stoll et al. ${ }^{37}$ provide an exampleby genetically mapping 239 phenotypic traits followed by pathway analysis (focusing on cardiovascular function in the rat). The large-scale profiling of segregating populations for many genes and many phenotypes can provide an even stronger approach in systems biology.

Perturbation strategies: pros and cons Statistical considerations. The genetical genomics approach involves perturbing many genes at the same time. These examples, illustrated in FIG. 1, show that multifactorial experimentation allows the study of many more biologically relevant questions than do strategies that perturb gene function in one factor at a time. Furthermore, in a multifactorial approach, the differential expression of two or more alleles is observed in a range of mixed genetic backgrounds. So, any gene the alleles of which show a clear expression difference over this range of backgrounds has a function and effect that is quite robust. The conclusions drawn from a genetical genomics experiment are more likely to apply to other genetic backgrounds than those of a knockout experiment. M oreover, the variance of the differential expression estimate is likely to be smaller for the genetical genomics than for the knockout strategy. With almost the same number of microarrays, and for almost the samearraying costs, the genetical genomics strategy increases the power to distinguish between real effects and noise.
In the genetical genomics approach, each segregant can becompared with the wild type on a separatemicroarray, as shown in FIG. 1b. However, costs can be reduced by the efficient allocation of material to multiplemicroarrays by, for example, using cyclic designs ${ }^{38}$. In a cyclic design, segregant 1 is compared with segregant 2 on microarray 1 , segregant 2 to segregant 3 on microarray 2 , and so on, doubling thenumber of times each segregant is profiled. If one geneis of particular interest and if it has two variants, such as RM and BY in the yeast exampledescribed above and in FIG. 1b, then segregants could be deliberately paired in such a way that each microarray contrasts an RM -allele-carrying segregant with a BY-allele-carrying segregant (leading to 40 replicates of differential expression between the RM and the BY allele). Clearly, thelayout of multifactorial experiments on multiple microarrays (or protein arrays) is another important level of multifactorial experimentation that would benefit from further research.

The principles of multifactorial experimentation laid out in this article can be traced back to the work in the early 1900s of thestatistician R. A. Fisher ${ }^{39}$ and are obvious to most people with statistical training. So, why are still so few multifactorial experiments being done? This is presumably due, in part, to the problems of drawing accurate inferences from data with a complex multifactorial structure; the statistical analysis of variance with multiple (interacting) factors might be perceived to be less straightforward. M oreover, the analysis of gene interactions requires adequate analysis methods to be developed. Advances in the area of analysis were discussed above.

Biological considerations. In the previous section, I compared the perturbation strategies simply on the basis of statistical arguments. O bviously, perturbation strategies should also be evaluated in the light of their biological pros and cons (TABLE 1). Genetic buffering is perhaps the simplest and most compelling example of how single-factor methods can fail ${ }^{40}$. For example, a biochemical network can buffer against the effects of a gene knockout: if compound $C$ can be produced through two routes, one catalysed by gene $a$, and the other catalysed by genes $b$ and $c$, then only a double knockout (genea and gene b or $c$ ) will lead to a loss-of-function phenotype for $C$ (BOX 2). Gene regulation is another example in which single-factor methods provide information, but cannot surpass multifactorial experiments. The heart of 
the regulatory mechanism is in the physical binding of trans-acting regulatory gene products to cis-acting binding sites of the genes regulated ${ }^{41,42}$ (BOX 2). Transcription factors are used typically at many times and places in the life cycle of an organism. The temporal and spatial uniqueness of transcription lies in the cis-acting modules of several binding sites. As transcription might require binding to all sites in a module, transcriptional regulation can be best studied through multifactorial experimentation.

Geneknockout, transgenesis and mutagenesis as strategies of experimental perturbation are applicable to model organisms, such as the mouse, yeast, Caenorhabditis elegans and Arabidopsisthaliana, but clearly not to humans. Genetical genomics can be applied to many organisms, but it is the only method available for studying systems biology in humans.

\section{Conclusions}

Biological and biomedical research will quickly advance from studying simple to complex traits, from using qualitative to quantitative biomolecular data, from carrying out separate to integrated analyses of different genomic and phenotypic data types, from analysing genes to gene interactions, and from sampling data from one or a few individuals to those from medium- to large-sized populations. Focused projects, such as the one aimed to generate a mouse population to be used for high-precision complex trait analysis (initiated by The Complex Traits Consortium), will markedly increase our knowledge of the complexity of biological traits and processes, and will teach us whether the "sky is the limit" holds for systems biology or not. The costs of microarray and related technologies have decreased to a level at which experiments with tens to hundreds of arrays have become affordable. It is now timely to begin genetical genomics experiments on a wider scaleand on larger populations, with the benefit of better power for analysing complex traits to their underlying multiple loci. Looking ahead, genomics, quantitative genetics and computing sciences will be integrated in a comprehensivestrategy of designing, modelling and analysing experimentsfor complex biological and biomedical systems: a new endeavour in the multidisciplinary field of bioinformatics.

Ritsert C. Jansen is at the Groningen Bioinformatics Centre, the Institute of Mathematicsand Computing Science, Faculty of Mathematical and Natural Sciences, and the Department of Medical Genetics,
Faculty of Medical Sciences, University of Groningen, PO Box 800, NL-9700AV Groningen, The Netherlands. e-mail: r.c.jansen@cs.rug.nl

doi: 10.38/nrg996

1. Chong, L. \& Ray, L. B. Whole-istic biology. Science $\mathbf{2 9 5}$ 1661 (2002).

2. Kitano, H. Systems biology. Science 295, 1662-1668 (2002).

3. Knowiton, R. G. et al. A polymorphic DNA marker linked to cystic fibrosis is located on chromosome 7. Nature 318, 380-385 (1985)

4. Kerem, B.-S. et al. Identification of the cystic fibrosis gene: genetic analysis. Science 245, 1073-1080 (1989).

5. Gilliam, T. C. et al. Localization of the Huntington's disease gene to a small segment of chromosome 4 flanked by D4S10 and the telomere. Cell 50, 565-571 (1987).

6. Huntington's Disease Collaborative Research Group. A novel gene containing a trinucleotide repeat that is expanded and unstable in Huntington's disease expanded and unstable in Huntington's dised
chromosomes. Cell 72, 971-983 (1993).

7. Hughes, T. R. et al. Functional discovery via a compendium of expression profiles. Cell 102, 109-126 (2000)

8. Ideker, T. et al. Integrated genomic and proteomic analyses of a systematically perturbed metabolic network. Science 292, 929-934 (2001).

9. Davidson, E. H. et al. A genomic regulatory network for development. Science 295, 1669-1678 (2002).

10. Ernest, $\mathrm{S}$. et al. Genetic and molecular control of folatehomocysteine metabolism in mutant mice. Mamm. Genome 13, 259-267 (2002).

11. J ansen, R. C. \& Nap, J. P. H. Genetical genomics: the added value from segregation. Trends Genet. 17, 388-391 (2001).

12. Brem, R. B., Yvert, G., Clinton, R. \& Kruglyak, L. Genetic dissection of transcriptional regulation in budding yeast. Science 296, 752-756 (2002).

13. Klose, J. et al. Genetic analysis of the mouse brain proteome. Nature Genet. 30, 385-393 (2002).

14. Wayne, M. L. \& McIntyre, L. M. Combining mapping and arraying: an approach to candidate gene identification. Proc. Natl Acad. Sci. USA $\mathbf{9 9}$ 14903-14906 (2002)

15. Demant, P. \& Hart, A. A. M. Recombinant congenic strains - a new tool for analyzing genetic traits determined by more than one gene. Immunogenetics $\mathbf{2 4}$ 416-422 (1986).

16. Fijneman, R. J . A., Ophoff, R. A., Hart, A. A. M. \& Demant, P. Kras-2 alleles, mutations, and lung tumor susceptibility in the mouse - an evaluation. Oncogene $\mathbf{9}$, 1417-1421 (1994).

17. Darvasi, A. Experimental strategies for the genetic dissection of complex traits in animal models. Nature Genet. 18, 19-24 (1998).

18. Nadeau, J. H., Singer, J. B., Matin, A. \& Lander, E. S. Analysing complex genetic traits with chromosome substitution strains. Nature Genet. 24, 221-225 (2000).

19. Threadgill, D. W., Hunter, K. W. \& Williams, R. W. Genetic dissection of complex and quantitative traits: from fantasy to reality via a community effort. Mamm. Genome 13, 175-178 (2002).

20. J annink, J L. \& J ansen, R. C. Mapping epistatic quantitative trait loci with one-dimensional genome quantitative trait loci with one-dimension
searches. Genetics 6, 337-342 (2001).

21. Gygi, S. P., Rochon, Y., Franza, B. R. \& Aebersold, R Correlation between protein and mRNA abundance in yeast. Mol. Cell. Biol. 19, 1720-1730 (1999).

22. J ansen, R. C., Nap, J. P. H. \& Mlynarova, L. Errors in genomics and proteomics. Nature Biotechnol. 20, 19 (2002).

23. Mather, K. \& I inks, J. L. Biometrical Genetics 3rd edn (Chapman \& Hall, London, 1982).

24. Brenner, S. et al. Gene expression analysis by massively parallel signature sequencing (MPSS) on microbead arrays. Nature Biotechnol. 18, 630-634 (2000).

25. Nap, J. P., Conner, A. J., Mlynarova, M., Stiekema, W. J. $\&$ J ansen, R. C. Dissection of a synthesized quantitative trait to characterize transgene interactions. Genetics $\mathbf{1 4 7}$ 315-320 (1997).

26. Mlynarova, L., Loonen, A., Mietkiewska, E., J ansen R. C. \& Nap, J. P. Assembly of two transgenes in an artificial chromatin domain gives highly coordinated expression in tobacco. Genetics 160, 727-740 (2002).

27. Ozbudak, E. M., Thattai, M., Kurtser, I., Grossman, A. D. \& van Oudenaarden, $A$. Regulation of noise in the expression of a single gene. Nature Genet. 31, 69-73 (2002).

28. Claverie, J. M. Gene number - what if there are only 30,000 human genes? Science 291, 1255-1257 (2001)

29. Carlborg, O., Andersson, L. \& Kinghorn, B. The use of genetic algorithm for simultaneous mapping of multiple interacting quantitative trait loci. Genetics 155 2003-2010 (2000)

30. Broman, K. W. \& Speed, T. A model selection approach for the identification of quantitative trait loci in experimental crosses. J. R. Statist. Soc. B 64, 1-6 (2002)

31. Ritchie, M. D. et al. Multifactorial-dimensionality reduction reveals high-order interactions among estrogenmetabolism genes in sporadic breast cancer. Am. J Hum. Genet. 69, 138-147 (2001).

32. Nelson, M. R., Kardia, S. L. R., Ferrell, R. E. \& Sing, C. F. A combinatorial partitioning method to identify multilocus genotypic partitions that predict quantitative trait variation. Genome Res. 11, 458-470 (2001).

33. Fijneman, R. J . A., de Vries, S. S., J ansen, R. C. \& Demant, P. Complex interaction of new quantitative trait loci, Sluc1, Sluc2, Sluc3, and Sluc4, that influence the susceptibility to lung cancer in the mouse. Nature the susceptibility to lung cance
Genet. 14, 465-467 (1996).

34. de Boer, M. P., ter Braak, C. J. F. \&J ansen, R. C. A penalized likelihood method for mapping epistatic quantitative trait loci with one-dimensional genome searches. Genetics 162, 951-960 (2002).

35. Wagner, A. How to construct a large genetic network from $n$ gene perturbations in fewer than $n^{2}$ easy steps. Bioinformatics 17, 1183-1197 (2001).

36. de la Fuente, A., Brazhnik, P. \& Mendez, P. Linking the genes: inferring quantitative gene networks from microarray data. Trends Genet. 18, 395-398 (2002)

37. Stoll, M. et al. A genomic-systems biology map for cardiovascular function. Science 294, 1723-1726 (2001).

38. Kerr, M. K. \& Churchill, G. A. Statistical design and the analysis of gene expression microarray data. Genet. Res. 77, 123-128 (2001).

39. Fisher, R. A. The Design of Experiments (Oxford Univ. Press, Oxford, UK, 1935).

40. Kitami, T. \& Nadeau, J. H. Biochemical networking contributes more to genetic buffering in human and mouse metabolic networks than does gene duplication. Nature Genet. 32, 191-194 (2002)

41. Yuh, C. H., Bolouri, H. \& Davidson, E. H. Genomic cis-regulatory logic: experimental and computationa analysis of a sea urchin gene. Science $\mathbf{2 7 9}$ analysis of a sea urchin

42. Fessele, S., Maier, H., Zischek, C., Nelson, P. J . \& Werner, $T$. Regulatory context is a crucial part of gen function. Trends Genet. 18, 60-63 (2002).

43. Frankel, W. N.\& Schork, N. J. Who is afraid of epistasis? Nature Genet. 14, 371-373 (1996).

44. Templeton, A. R. in Epistasis and the Evolutionary Process (ed. Wolf, J . B.) 41-57 (Oxford Univ. Press, Oxford, UK, 2000).

Acknowledgements

This article is dedicated to my former room-mate and bioinformatics colleague J. (Hans) M. Sandbrink, who recently passed away. I am grateful to M. P. de Boer for carrying out the simulations presented in Box 1, to R. W. Williams for providing early access to his mouse work, and to three reviewers for their constructive comments.

\section{(4) Online links}

\section{DATABASES}

The following terms in this article are linked online to: OMIM: http://www.ncbi.nlm.nih.gov/Omim

cystic fibrosis | Huntington disease

Saccharomyces Genome Database:

http://genome-www.stanford.edu/Saccharomyces

GAL7 | GAL10 |XBP1 |YLL007C

\section{FURTHER INFORMATION}

List of gene and QTL mapping software:

http://linkage.rockefeller.edu/soft

RitsertJ ansen's laboratory: http://www.cs.rug.nl/ ritsert

Ritsert J ansen's laboratory: $h$ ttp://www.cs.rug.nl/ ritsert

Access to this interactive links box is free online. 